\title{
REFLEXIONES SOBRE EL ESTADO ACTUAL DE LA ÉTICA EN INVESTIGACIÓN EN ARGENTINA
}

\author{
Delia Outomuro*
}

\begin{abstract}
Resumen: Los propósitos de este trabajo son: 1) analizar el estado actual de la ética en investigación en Argentina a partir de observaciones efectuadas por nuestro grupo en los últimos años en los reglamentos de publicación de revistas médicas nacionales y a partir de una reflexión crítica de la legislación vigente sobre el tema; 2) proponer algunas estrategias innovadoras que apunten a garantizar la corrección ética de las investigaciones. Entre ellas destacamos: 2.a) la independencia de los Comités de Ética en Investigación (CEI); 2.b) la necesidad de estandarizar, homologar, acreditar y auditar los CEI; 2.c) la necesidad de actualizar las normas CIOMS 1991 considerando no sólo los posibles daños biológicos, sino también los psicológicos, socioculturales y legales; 2 .d) el requerimiento de "certificados de conformidad bioética" para la publicación de las investigaciones; 2.e) la incorporación de las tareas del CEI dentro de los procesos llamados "sistemas de calidad".
\end{abstract}

Palabras clave: Bioética, investigación, comités, ley, revistas científicas.

\section{CONSIDERATIONS ABOUT THE CURRENT STATE OF ETHICS IN RESEARCH IN ARGENTINE}

\begin{abstract}
The purposes of this paper are: 1) to analyze the current state of ethics in research in Argentine from the observations made by our group in national research magazines and from a critic review of the law; 2) to put forward some new strategies focused in making research secure and ethical, and especially 2.a) the independence of REC; 2.b) the need for standards, vouching for, accreditation and auditing REC; 2.c) the need for reviewing CIOMS 1991 norms taking into account not only biological risks but also psychological, social, cultural and legal ones; 2 .d) the requirement of certificates of bioethical qualification to publish the results of a research; 2.e) the incorporation of the REC tasks into the processes called "quality systems".
\end{abstract}

Key words: Bioethics, research, committees, law, scientific magazines

\section{REFLEXÕES SOBRE O ESTADO ATUAL DA ETICA NA PESQUISA NA ARGENTINA}

Resumo: Os objetivos deste trabalho são: 1) analisar o estado atual da ética em pesquisa na Argentina a partir de observações efetuadas por nosso grupo nos últimos anos, nos regulamentos de publicações de revistas médicas nacionais e a partir de uma reflexão crítica da legislação vigente sobre o tema; 2) propor algumas estratégias inovadoras que garantam a retidão ética das pesquisas. Entre outras destacamos: 2.a) a independência dos comitês de ética em pesquisa; 2.b) a necessidade de padronizar, homologar, acreditar e auditar os comitês de ética em pesquisa; 2.c) a necessidade de atualizar as normas CIOMS 1991 considerando não somente os possíveis danos biológicos mas também os danos psicológicos, socio-culturais e legais; 2.d) a exigência de "certificação conformidade bioética para a publicação das pesquisas; 2.e) a incorporação das tarefas do Comitê de Ética em Pesquisa no contexto dos processos denominados de "sistemas de qualidade".

Palavras chave: Bioética, pesquisa, comitês, lei, revistas científicas

Profesora Adjunta y Coordinadora de la Unidad Académica de Bioética, Facultad de Medicina, Universidad de Buenos Aires.

Correspondencia: doutomuro@fmed.uba.ar 


\section{Introducción}

Hoy, más que nunca, se insiste en la necesidad de respetar las normas éticas cuando se investiga. Sin embargo, la experiencia nos muestra que, una vez más, hay una gran brecha entre el decir y el hacer, y que aquello que se sostiene discursivamente no siempre encuentra su correlato en la praxis.

Esta situación es particularmente preocupante en países emergentes donde suelen llevarse a cabo grandes estudios epidemiológicos, así como ensayos farmacológicos en distintas fases patrocinados por los países centrales. Distintas características de la población de dichos países periféricos (pobreza, analfabetismo, desinformación, entre otros) se conjugan para hacer vulnerables a los miembros de estas comunidades. Asimismo, la legislación de estos países suele ser bastante permisiva, o bien, si no lo es, la deficiencia en los mecanismos de control hacen que, en la práctica, esta permisividad sea un hecho.

Mi tesis establece que, en este contexto, los comités de ética (CE), tal como están funcionando, no brindan suficiente protección a los sujetos de investigación. Esto hace necesario un replanteo del tema así como el establecimiento de tácticas y estrategias que permitan su consolidación y su papel protector.

En este trabajo pretendo: 1) analizar el estado actual de la ética en investigación en Argentina a partir de observaciones efectuadas por nuestro grupo en los últimos años en los reglamentos de publicación de revistas médicas nacionales y a partir de una reflexión crítica de la legislación vigente sobre el tema; 2) proponer algunas estrategias innovadoras que apunten a garantizar la corrección ética de las investigaciones.

\section{Los Comités de Ética. Una diferencia significativa}

Como es sabido, existe una sustancial diferencia entre los Comités de Ética en Investigación (CEI) y los Comités de Ética Clínica (CEC). Sin embargo, esto no está reproducido en la legislación argentina. Una de las funciones de los CEC es la consultiva (aconsejar) y sus decisiones no son obligatorias; los CEC no son jueces ni sus decisiones, órdenes. Éste es un punto importante en la diferencia con los CEI. Estos últimos se encargan de evaluar protocolos de investigación y su dictamen, en relación con la ponderación ética de dicho protocolo, es decisorio para la viabilidad de un estudio científico. Existe aún cierta controversia acerca de hasta dónde el CEI debe extender su control; si debe, por ejemplo, ejercer algún tipo de monitoreo en el curso de la investigación. Sin embargo, hay un consenso claro en su poder de veedor de la adecuación ética de los protocolos.

Ambos comités deben ser, sin embargo, multidisciplinarios. En el caso de los CEI, una composición dominante de médicos o científicos podría sesgar el análisis en dirección de un consenso previo - tal vez implícito- orientado hacia el desarrollo tecnocientífico haciéndolo prevalecer sobre otros valores.

\section{La investigación en países emergentes. Aproximación a su problemática ética}

Muchos países emergentes carecen de un marco regulador que proteja a los sujetos de investigación y si, en el mejor de los casos, este marco existe, suele ser laxo o carecer de efectividad en su aplicación. No es casual que los países centrales elijan estas latitudes para esos fines(1). Conviene recordar aquí la clásica distinción entre "legalidad" (conformidad con el derecho positivo) y "legitimidad" (conformidad con la ética) y que no todo lo legal es legí- 
timo. El hecho de que el marco legal de un país periférico permita llevar a cabo una investigación no es condición suficiente para realizarla, si ella carece de legitimidad.

Los principios y reglas bioéticas son frecuentemente violados de diversas maneras, algunas veces de forma abierta, como cuando se omite el consentimiento de los sujetos de investigación; otras, de modo más solapado. Tal es el caso de consentimientos obtenidos en un idioma distinto del que habla el sujeto. El principio de justicia suele ser también vulnerado. Muchas veces las muestras no son obtenidas equitativamente de modo tal que se compartan riesgos y beneficios. Cuando la investigación se lleva a cabo en la población de países emergentes habrá que tener en cuenta si lo que se está investigando es o no de interés para dicha población y, en caso de serlo, si tendrá acceso a los potenciales beneficios. Son bien conocidos los estudios que prueban, por ejemplo, drogas oncológicas en comunidades donde la patología en cuestión no es el principal problema de salud. La conflictividad se incrementa cuando el estudio pretende ser randomizado. En este caso, la Declaración de Helsinki intenta la protección de los sujetos al sostener que el grupo control debe recibir el mejor tratamiento probado a la fecha. Pero esto no resuelve el problema, porque ¿a qué "mejor tratamiento" se refiere? ¿Al aplicado corrientemente en la comunidad emergente? ¿O al aplicado en el país central que actúa como patrocinador? Recuérdese la discusión suscitada en torno a la investigación farmacológica en embarazadas con SIDA realizada en África y Asia ${ }^{1}$.

En 1997 se investigó la dosis mínima de AZT necesaria para prevenir la transmisión vertical del HIV. Para ello se utilizaron mujeres embarazadas HIV positivas del Sahara y del sudeste asiático, las que fueron distribuidas en dos grupos. Uno de ellos recibió la dosis investigada y el otro recibió placebo. Los autores argumentaron a su favor que el grupo control había recibido el mejor tratamiento utilizado en su comunidad, es decir, nada. Por otra parte, el grupo experimental se había beneficiado al recibir la dosis en prueba de AZT.
También, en torno al principio de justicia, se plantea el problema de la eticidad de la Fase I de la investigación clínica farmacológica. En esta fase se aplica la droga en estudio a personas que no poseen la enfermedad para la cual fue concebida. Por lo tanto, estas personas están corriendo riesgos innecesarios en la medida en que no son ellos quienes recibirán los beneficios del fármaco:

"La determinación de cuándo los riesgos potenciales a sujetos individuales exceden el potencial de los beneficios a la sociedad acarrea comparaciones interpersonales que, tanto conceptual como prácticamente, son muy difíciles y se acercan mucho a la explotación. Aunque tal comparación es inherente a toda investigación clínica, es particularmente exagerada en la Fase I de la investigación, durante la cual no se espera ningún beneficio para el individuo [...] no existe un marco determinado sobre cómo "balancear" los beneficios sociales contra los riesgos individua$\operatorname{les}(2)$ '.

La desprotección de las personas en países periféricos se evidencia también en la Fase IV, cuando la droga está ya comercializada, al fallar los mecanismos de farmacovigilancia. Poco antes de realizar este trabajo, la opinión pública argentina se conmovió ante una noticia difundida por los medios periodísticos. Según un informe del diario The New York Times, la unidad Bayer, Cutter Biological, siguió comercializando Factor VIII que conllevaba un alto riesgo de transmisión de HIV en países de América Latina y de Asia cuando ya no lo hacía ni en Estados Unidos ni en Europa ${ }^{2}$. Al parecer, en 1985, la FDA de los Estados Unidos amonestó

Bogdanich W, Koll E. Vendian en Argentina un remedio infectado con sida. The New York Times. Especial para el diario Clarín. Buenos Aires, 23 de mayo de 2003. 
a Cutter y a otras tres compañías que actuaron de modo semejante y sostuvo que "es inaceptable vender estos productos a otros países", pero pidió que el asunto fuera "resuelto silenciosamente, sin alertar al Congreso, a la comunidad médica y al público"3. Otro informe periodístico da cuenta de que se siguen vendiendo en Argentina drogas como la clozapina, la terfenadina y el astemizol cuya venta ha sido prohibida en Estados Unidos ${ }^{4}$. Es que, según la industria farmacéutica, poner en el mercado una nueva molécula cuesta unos 500 millones de dólares y, si a los seis meses hubiera que retirarla por efectos adversos, las pérdidas serían cuantiosas $^{5}$.

\section{Estado actual de la ética en investigación en Argentina. Algunas repercusiones periodísticas, a modo de ejemplo}

En la edición del 27 de mayo de 2003 del diario Clarín se lee:

"Según lo denuncia un informe sobre ética en investigaciones farmacológicas con pacientes elaborado por la Defensoría del Pueblo de la Nación, el Estado no controla debidamente y con eficacia la experimentación con drogas realizada en personas que padecen cáncer [...] se hallaron 17 casos en los cuales los médicos -tanto particulares como de hospitales públicos y centros asistenciales privados-habían realizado investigaciones con drogas sin autorización. Los pacientes no fueron informados de la investigación a la que eran sometidos, no se les requirió su consentimiento ni tampoco se les pagó el tratamiento".

Ríos SA. Dolor de cabeza para Bayer. Denuncia de The New York Times. Diario La Nación. Buenos Aires, 23 de mayo de 2003.

4 La venta de estos fármacos fue prohibida en Argentina recién en agosto de 2003.

Kiningsberg Y. Prohibidas en EE.UU., pero aceptadas acá. Clarín. Buenos Aires 23 de mayo de 2003.
La edición del 29 de mayo de 2003 del diario Los Andes de Mendoza publica:

"En el país se efectuaron 17 investigaciones no autorizadas. Estas investigaciones clandestinas salieron a la luz durante el Congreso de la Sociedad Americana de Oncología Clínica (ASCO) en 2001 y 2002. Allí, un grupo de médicos e institutos de todo el país publicaron resúmenes de diferentes estudios realizados en Argentina (26 investigaciones en total, todas en pacientes con cáncer). De éstas, 17 no se habían notificado ni aceptado por el ANMAT'.

\section{Un diagnóstico a partir de las publicaciones médicas}

Lamentablemente, no son muchas las investigaciones médicas publicadas que manifiestan, explícitamente, respetar los lineamientos de la ética en investigación. Nuestro grupo ha investigado los datos presentes en el reglamento de algunas revistas médicas argentinas en relación con la exigencia de normas éticas para la publicación de investigaciones hechas con seres humanos. Observamos que: 1) el 33,3\% no contenía ningún tipo de indicación para los autores; 2) en el $57,9 \%$ de las que sí tenían reglamento de publicación, el mismo se limitaba a observaciones de orden técnico, y 3) sólo el $16 \%$ del total de revistas consultadas incluía pautas éticas ${ }^{6}$.

Como vemos, son escasas las publicaciones médicas argentinas que reparan en estos límites, al menos en la muestra por nosotros estudiada. El respeto por la autonomía es uno

Outomuro D, Bortz JE, Sabio F, Kohn Loncarica AG. Requisitos éticos para la publicación de trabajos realizados con seres humanos en algunas revistas argentinas. VI World Congress of Bioethics "Power and Injustice". (Unjustice) Brazilian Society of Bioethics. Brasilia, 30 de octubre al 3 de noviembre de 2002. 
de los aspectos que suele considerarse. En este sentido, el valor más resguardado suele ser la privacidad, en especial en relación con la publicación de fotografías para lo cual se solicita el consentimiento del paciente. Consideramos que esto es condición necesaria, pero no suficiente para garantizar la moralidad del trabajo. Los principios de no maleficencia, de beneficencia y de justicia han de ser, también, tenidos en cuenta. Es decir, a pesar de que el paciente autorice publicar una foto que pudiera identificarlo, el investigador debe velar por el cumplimiento de los principios citados. Llamativamente, muchas revistas aceptan la publicación de fotos exigiendo sólo el permiso del paciente cuando lo que corresponde es que la fotografía presente bandas enmascaradoras (por ejemplo, tapado de ojos).

Con respecto a la evaluación por parte de un comité de ética, no suele exigirse que sea realizada por un comité independiente que garantice la objetividad del análisis. En nuestro país existe un organismo, la Administración Nacional de Medicamentos, Alimentos y Tecnología (ANMAT), encargado de fiscalizar la realización de protocolos de investigación destinados a la aprobación de un nuevo fármaco, una nueva indicación de un fármaco ya aprobado o una nueva presentación de un fármaco ya aprobado. Podemos afirmar que esta entidad toma recaudos en cuanto a la calidad ética de los estudios. Sin embargo, estimaciones aún no publicadas, recogidas por nuestro grupo, establecen que los ensayos destinados a cualquiera de los tres fines mencionados representan sólo entre el 10 y el 20\% de los estudios clínicos. Esto significa que entre el 80 y el $90 \%$ de los trabajos de investigación médica que involucran a sujetos humanos, en su mayoría pacientes, están fuera de la esfera de competencia de la ANMAT y de su control ético exigido a través de la disposición 5330 de aprobación de productos farmacéuticos comerciales $^{7}$ (3).

\section{Análisis de la legislación vigente}

Presentaremos ahora algunas leyes y normas reguladoras que existen en nuestro país con el fin de obtener una visión general de la situación argentina con respecto a los Comités de Ética y a su diferenciación.

\section{Decreto 426/98. Comisión Nacional de Ética Biomédica}

Creada en 1998 por el Ministerio de Salud y Acción Social con el fin de contar con una entidad asesora en temas de bioética. Entre las razones y fundamentos para crear esta comisión no se menciona el área de investigación biomédica. Refiere que "el avance tecnológico en las ciencias biológicas y médicas ocasiona [...] problemas éticos, morales y legales" y prevé estos problemas en torno "al proceso de toma de decisiones en materia de salud, con la asignación de recursos escasos y la interpretación operativa de los conceptos de equidad, solidaridad, eficacia y calidad de los servicios".

Esta comisión corresponde a los comités que dependen de una instancia nacional. Entre los fundamentos de su creación sólo se mencionan las funciones propias de un CEC y no se hace referencia alguna a la necesidad de control ético de la investigación biomédica ni a la posibilidad de crear o promover instancias con tal fin. Explícitamente, en el Art. 1, inciso d, se plantea como objetivo "promover que en todas las instituciones de salud se organicen y funcionen Comités de Ética Biomédica”.

Bortz JE. Certificados de Conformidad Bioética. Segundas Jornadas Nacionales de Ética Biomédica. Buenos Aires: Ministerio de Salud y Acción Social. Organización Panamericana de la Salud. Organización Mundial de la Salud; 30 de noviembre y $1^{\circ}$ de diciembre de 2000. (En prensa). 


\section{Ley Nacional 24742/96}

En su Art. 1 establece "que en todo hospital del sistema público de salud y seguridad social, en la medida que su complejidad lo permita, deberá existir un Comité Hospitalario de Ética, el que cumplirá funciones de asesoramiento, estudio, docencia y supervisión de la investigación respecto de aquellas cuestiones éticas que surjan de la práctica de la medicina hospitalaria". El Art. 2 menciona una conformación interdisciplinaria sin aclarar este concepto y establece que la actividad del comité será dependiente de la dirección del hospital. Consideramos que la "independencia" de cualquier comité de ética es un requisito fundamental para evitar conflictos de interés. El Art. 3 menciona 14 temas sobre los que versará su actividad, uno de ellos es la "experimentación en humanos", los restantes corresponden a la ética clínica. El Art. 4 establece que las recomendaciones del comité "no tendrán fuerza vinculante", lo cual no se compadece con lo esperable para un CEI.

No se marcan diferencias entre los CEC y los CEI. Las funciones que se le atribuyen son las de un CEC y, si bien se menciona el área de investigación, la expresión "supervisión de la investigación" es demasiado vaga e inespecífica en cuanto a la función que debería cumplir un comité a este respecto.

Municipalidad de General Pueyrredón. Ordenanza 10886/96. Creación de un comité municipal de bioética

Entre las consideraciones que justifican la creación del comité, se cita la "necesidad de contar con asesoramiento altamente especializado en el análisis de temas relacionados con la bioética y el desarrollo tecnocientífico en el campo de las ciencias de la vida y la atención de la salud". También se sostiene que es menester adoptar "nuevas instancias de reflexión, asesoramiento y control éticos" en el ámbito de la investigación con seres humanos.

Aunque se menciona el control ético de la investigación con humanos como uno de los fundamentos de su creación, el Comité tiene las funciones propias de un CEI: "funciones educativas, investigativas, consultivas y de asesoramiento para la resolución de posibles conflictos de interés" (Art. 4); "su consulta será optativa y sus recomendaciones no serán vinculantes" (Art. 5). Recordemos que los comités encargados de evaluar la ética de las investigaciones biomédicas tienen poder de veto.

Por otra parte, el Art. 3 establece el carácter interdisciplinario del comité; pero, inmediatamente, describe una conformación en la que "la mitad más uno de sus integrantes serán profesionales de las ciencias de la vida y de la salud" con lo cual la interdisciplinariedad resulta meramente retórica.

\section{Provincia de Jujuy. Ley $4861 / 95$ Creación de Comités Hospitalarios de Ética}

Se propone la creación de Comités Hospitalarios de Ética "cuya labor es exclusivamente de asesoramiento y docencia sobre dilemas que surgen durante la práctica de la medicina hospitalaria involucrados en decisiones éticas en la investigación, prevención y tratamiento de las enfermedades de las personas" (Art. 2). Nuevamente se confunden los roles. La función es exclusivamente consultiva y educativa, incluso en decisiones éticas en la investigación.

La mentada interdisciplinariedad es también retórica, pues su conformación se asemeja a un comité de "notables" designados por el director del hospital, debiendo ser médicos el 50\% de sus miembros (Art. 3 y 4). 
Provincia de Tucumán. Ley 6507/93 Creación de Comités Hospitalarios de Ética

Caben las mismas consideraciones hechas para la ley 4861/95 de la provincia de Jujuy.

\section{Provincia de Río Negro. Ley 3099/97 Salud Pública. Bioética en Investigación, Análisis y Difusión}

Hace referencia a la creación de un Comité Provincial de Bioética entre cuyas funciones se menciona la de "fomentar la conformación de Comités Hospitalarios de Bioética". Tales comités cumplirán la función de asesorar sobre los dilemas éticos que ocurran dentro de la institución. El Art. 10 cita cuatro áreas temáticas de su incumbencia: ética de la salud pública, ética clínica, educación en bioética y ética de la investigación. No obstante, no se establecen funciones reguladoras de la investigación biomédica. La descripción de esta área resulta confusa. Su mención pareciera referirse a la recomendación de impulsar temas especiales de interés.

\section{Provincia de Buenos Aires. Ley 11044/91}

En el Capítulo 1, Título 2, establece que las investigaciones "deben ser aprobadas por los comités de ética y de investigación del establecimiento o institución de salud. En toda institución de salud con funciones de investigación deberán funcionar en forma continua un comité de ética y un comité de investigación”. En el Título 4 del mismo capítulo, Art. 36, señala que sus funciones serán: “a) asesorar al responsable de la institución sobre la autorización de investigaciones, sobre la base del protocolo de investigación y de la supervisión directa; b) asistir al equipo de investigación, y c) controlar la aplicación de esta ley y su reglamentación".

Si bien esta ley es, dentro de las aquí anali- zadas, la que más detalla las características que debe tener la investigación en los servicios asistenciales de la provincia, denomina "comité de ética" a lo que sería un CEI y no diferencia con claridad entre CEI y CEC. Establece sí una distinción entre comité de ética y comité de investigación, pero este último estará abocado a evaluar los aspectos metodológicos del proyecto.

Ciudad Autónoma de Buenos Aires. Ley 153 Ley Básica de Salud de la Ciudad de Buenos Aires

En el Art.14, entre los objetivos del Subsector Estatal de Salud, se menciona: "inciso k) establecer la creación de comités de ética en los efectores". En el Art. 38, correspondiente al Capítulo de Docencia e Investigación, se remite a la Legislatura el proyecto de creación de un consejo de investigación de salud, como organismo de conducción y coordinación de la actividad de investigación en el sistema de salud y, entre sus lineamientos, incluye, en el Art. 39, la constitución de "una instancia de normatización y evaluación ética de la investigación".

En esta norma se especifica la necesidad de evaluación ética de la investigación y se menciona una instancia de normatización de la investigación, pero no la distingue como CEI.

\section{Ciudad Autónoma de Buenos Aires. Resolución $N^{\circ} 1154 / 03$}

El 9 de junio de 2003, y en el marco de la Ley 153, por la citada resolución se crea el Comité Central de Bioética en Investigación en Salud. Su propósito es proteger los derechos de las personas que participen en proyectos de investigación, promover la capacitación de los investigadores y brindar resguardo ético a los investigadores y a las instituciones en que se desarrollen dichos proyectos 
$\left(\text { Art. } 3^{\circ}\right)^{8}$. Este comité tiene, entre sus funciones, supervisar, evaluar y emitir dictámenes sobre los proyectos de investigación desarrollados en los hospitales dependientes del gobierno de la Ciudad de Buenos Aires.

Con esta resolución, sin duda, se ha dado un importante paso en ética de la investigación. No obstante, al momento de redactar este artículo, este comité está recién elaborando su reglamento interno y debatiendo distintos aspectos que tienen que ver, entre otras cosas, con la forma en que llevará a cabo su tarea en relación con los CEC de los hospitales. Éstos, junto con los Comités de Docencia e Investigación, son los encargados, hasta el momento, de la evaluación de los protocolos.

\section{Hacia una investigación científica con responsabilidad. Tácticas y estrategias propuestas}

Con el objeto de revertir esta situación y tender hacia una investigación en un marco de responsabilidad, nuestro grupo ha delineado diferentes estrategias. Destacamos las siguientes:

\section{A) Garantizar la corrección metodológica}

Fernando Lolas Stepke propone distinguir entre legalidad y legitimidad en el análisis ético de la investigación clínica:

"Legalidad se refiere al cumplimiento de procedimientos aceptados por la comunidad científica relativos a la validez, confiabilidad y solvencia del trabajo de investigación. Legitimidad alude a la propiedad con que en el contenido de la publicación se respetan los principios éticos(4)".

En opinión de la autora, este último propósito no debería ser más que un "efecto secundario o colateral" del verdadero propósito de un CEI: proteger a las personas en su papel de sujetos de investigación.
Sostenemos que el cumplimiento de las reglas metodológicas es el primer requisito ético. Un estudio que no resista la crítica epistemológica es ipso facto no ético, pues no tiene sentido someter a riesgo, por mínimo que éste sea, a un ser humano cuando los resultados de la investigación serán dudosos por fallas metodológicas. Se debe destacar que, según un estudio publicado en el Journal of American Medical Association (JAMA), sólo el 50\% de los trabajos presentados en congresos médicos fue publicado en revistas de prestigio durante los tres años siguientes. Ello es atribuido, por los autores del estudio, a su falta de rigor metodológico que "no resisten el filtro de una publicación de prestigio [mientras que] los organizadores de congresos no son demasiado exigentes con los trabajos, porque quieren garantizar una gran participación"(5).

B) Identificar y disolver los conflictos de intereses

La legalidad de un proyecto se refiere, tanto a los aspectos epistemológicos y metodológicos de la investigación cuanto a lo que se ha dado en llamar la ética profesional del investigador (honestidad intelectual, trabajo en equipo, reconocimiento del mérito de los colegas, etc.). Así:

"Un estudio puede ser inobjetable desde el punto de vista de la legalidad, pero inaceptable en cuanto a la legitimidad. La distinción entre ambas dimensiones es, por cierto, artificial. Ningún estudio mal concebido o insuficientemente elaborado puede pasar un examen ético. Un conflicto no declarado de intereses (por ejemplo, el informe tendencioso de un investigador sobre un fármaco, de cuya empresa fabricante es accionista) vulnera la legalidad y la legitimidad en un solo acto(4, p. 290)". 
C) Revisar y actualizar las normas CIOMS 1991

En los estudios epidemiológicos se manifiesta, quizás con mayor claridad que en los biomédicos, el conflicto entre derechos del individuo y derechos de la sociedad. En nuestra opinión, y desde una perspectiva kantiana, ninguna persona puede ser considerada un medio para un fin, ni siquiera para un fin loable como puede serlo el conocimiento científico(6). Proponemos su revisión desde esa perspectiva y sugerimos tener en cuenta los siguientes puntos:

1. Los posibles daños en los estudios epidemiológicos son: biológicos, psicológicos, socioculturales y legales. Cualesquiera de ellos puede ser lo suficientemente importante como para justificar la obtención del consentimiento informado.

Las normas CIOMS afirman que los estudios observacionales "no exigen procedimientos invasivos más allá de las preguntas que se hacen" y que "estos tipos de estudios suponen riesgos mínimos para los participantes”. En nuestra experiencia estas consideraciones minimizan los riesgos de dichos estudios. Creemos que los daños que puede sufrir un sujeto de investigación exceden el plano biológico y que han de tenerse también en cuenta los riesgos psicológicos, sociales y legales. De acuerdo con el marco social o legal imperante estos riesgos pueden ser incluso más importantes que los físicos. Valga como ejemplo un estudio que investigue antecedentes de aborto en países donde éste es ilegal.

Asimismo, muchas enfermedades son estigmatizantes. Quizás el SIDA represente un caso paradigmático en este sentido, pero cada sociedad o cultura construye significados distintos en torno a las afecciones por lo que la potencialidad discriminatoria o estigmatizante de una enfermedad ha de entenderse culturalmente y no de manera universal.
2. La autorización de un estudio por parte del líder comunal podrá ser condición necesaria, pero no suficiente para realizar dicho estudio, debiéndose obtener el permiso de las personas involucradas.

3. CIOMS 91 reconoce el carácter "poco ético" de los estudios randomizados, consideración que compartimos. Sin embargo, no se habla del uso de placebos. Los estudios randomizados en los que el grupo control recibe placebo siguen siendo frecuentes. En ellos son varias las reglas y principios bioéticos que pueden violarse por lo que, en general, se considera que ha de tratarse de estudios excepcionales que sólo se justifican si se cumplen ciertas condiciones. E1 engaño no está justificado a excepción de que el conocimiento que se desea obtener sea relevante, que no se pueda obtener de otro modo, que los sujetos involucrados estén al tanto de que la información que se les dará será parcial (y consientan en ser engañados) y que no sean expuestos a riesgos considerables(7).

4. El marco legal de los países emergentes donde se realiza una investigación patrocinada por países centrales ha de tenerse en cuenta, pero que dicho marco permita llevar a cabo el estudio, no significa ipso facto que sea legítimo. El análisis ético nunca deberá omitirse.

5. Destacamos la participación de los comités de ética en investigación también en el análisis de todos los protocolos epidemiológicos. Con el propósito de evitar los conflictos de intereses, entendemos que estos comités deben ser independientes de los investigadores, patrocinadores o instituciones en donde se realice el estudio. Asimismo, resaltamos la importancia de la presencia de miembros representativos de la comunidad. 
6. Las apreciaciones sobre el principio de no maleficencia deberían ampliarse. No sólo hay que tener en cuenta los daños "evitables", sino más bien los "previsibles" (sean evitables o no). Asimismo, debería aclarase que el daño ocasionado no siempre tiene que ver con "acciones", sino muchas veces con "omisiones". Es más, en ciertas ocasiones una omisión puede acarrear más daños que una acción( $7, p p$. 219-225). Creemos que esto es relevante en el caso de los estudios epidemiológicos prospectivos. La simple observación, el no hacer nada más que observar no pone a salvo la no maleficencia. Recuérdese el caso Tuskegee en el que los investigadores simplemente observaban la evolución natural de la sífilis, sin intervenir siquiera cuando la penicilina podía aportar una esperanza.

7. Son precondiciones para la aprobación ética de cualquier protocolo la aptitud del investigador principal y de su grupo, y la calidad metodológico-científica del estudio.

8. En líneas generales, CIOMS 91 descansa en la conducta ética y prudencia del investigador. Pero la toma de decisiones acerca de si se justifica o no violar alguna regla o principio ético en un caso particular no puede dejarse librada a los investigadores por ser éstos parte interesada.

9. Cuando se recogen datos para las historias clínicas asistenciales, habría que preguntar al paciente si autoriza que dicha información sea utilizada para investigaciones científicas, dejándose clara constancia de su autorización o negativa. Insistimos en la necesidad de obtener el consentimiento en los estudios experimentales y en los observacionales prospectivos. En el caso de los retrospectivos y los de corte transversal, cuando se basan en historias clínicas, los pacientes deberían informar en el momento de su confección si autorizan que, en el futuro, sus datos sean utilizados con fines de investigación. Es necesario recordar que, cuando el paciente acude al médico tratante, consiente que se obtenga información sobre él con el propósito de que la misma redunde en su beneficio personal. Por el contrario, en una investigación el objetivo principal no es beneficiar al paciente, sino obtener información. Los datos cuya obtención fue autorizada por el paciente con un propósito no pueden ser utilizados para otro.

10.Los "trabajadores asalariados" tendrían que incluirse en la categoría de grupos vulnerables. En los exámenes preocupacionales, así como en los exámenes periódicos de salud, los trabajadores pueden ser coaccionados para consentir prácticas que, en otro contexto, no autorizarían.

D) Normalizar los comités de ética en investigación

En este punto proponemos:

1. Modificar la legislación vigente distinguiendo entre CEC y CEI y estableciendo, claramente, el carácter "vinculante" de sus resoluciones.

2. Garantizar la independencia de los CEI. Las investigaciones realizadas en una institución no deberían ser evaluadas sólo por un comité que pertenezca a la misma institución, pues este proceder no garantiza la resolución de posibles conflictos de intereses. De ahora en adelante nos referiremos a los CEI como CIEI (Comité Independiente de Ética en Investigación). 
3. Establecer una conformación que garantice la correcta evaluación del proyecto ${ }^{9}$.

\section{E) Auditar y acreditar los CIEI}

Consideramos este punto de fundamental importancia en las evaluaciones de los proyectos para garantizar su calidad y, por lo tanto, la de su producto. Dicha auditoría podría realizarse de varias maneras. Una forma es el acuerdo de trabajo entre comités de ética, una especie de auditoría "circular" o "cruzada".

Sin embargo, entendemos como ideal el control de calidad efectuado por un comité nacional de bioética y/o por organismos supranacionales de reconocido prestigio en el tema. Para ello será necesario contar con criterios de evaluación oportunamente establecidos por parte de estos organismos. Lo que proponemos es algo semejante a los mecanismos de acreditación que ya existen en numerosos países, por ejemplo, para acreditar universidades. En Argentina se realiza a través de la Comisión $\mathrm{Na}$ cional de Educación y Acreditación Universitaria (CONEAU).

\section{F) Exigir "Certificados de Conformidad Bioética"}

Si se trata de un estudio farmacológico se infiere que deberá haber un experto en investigación farmacológica entre los integrantes del comité. Pero este experto no tendrá nada que hacer si el protocolo se refiere a una investigación sociológica, requiriéndose, en este caso, un sociólogo. Por lo tanto, en un CIEI habrá miembros permanentes y miembros convocados ad hoc. Citamos como ejemplo el CIEI de la Facultad de Medicina de la Universidad de Buenos Aires. Este CIEI fue creado por la Resolución (CD) 1658/01 y su reglamento interno fue aprobado por Resolución (CD) 2516/02. En este reglamento se establece que el comité estará conformado por nueve miembros: un presidente, un vicepresidente, un secretario o coordinador y seis vocales permanentes. Estos miembros permanentes son: un filósofo, un abogado, un médico legista, un investigador clínico, un investigador básico (farmacólogo) y un lego como representante de los sujetos de investigación. Asimismo, se establece que habrá miembros convocados como asesores de acuerdo con las características del estudio. Estos asesores serán sociólogos, antropólogos, psicólogos, comunicadores sociales, veterinarios, paramédicos u otros expertos en la materia del proyecto de investigación.
Como vimos, existe en Argentina un organismo estatal de control y regulación llamado ANMAT, dependiente del Ministerio de Salud de la Nación (sucesor del viejo Instituto Nacional de Farmacología y Bromatología). Pero el espectro de su competencia en los ensayos clínicos es el nuevo fármaco, la nueva presentación, la nueva indicación.

También afirmamos que nuestros datos aún inéditos permiten arriesgar que entre el 80 y el 90\% (cifras estimadas merecedoras de estudios estadísticos serios) de los trabajos hechos con personas no están destinados a un nuevo fármaco, a una nueva presentación o a una nueva indicación. Por lo tanto, no están bajo la esfera de competencia del ANMAT. La normativa vigente no requiere que sean reportados a ningún organismo oficial y su destino final suele ser una reunión científica o una publicación.

Ahora bien, hemos analizado los requisitos que los comités científicos exigen para la aceptación de comunicaciones libres y hemos revisado las condiciones, en busca de la exigencia de requisitos éticos, para salvaguarda de los derechos de los seres humanos sometidos a proyectos de investigación. Los resultados preliminares indican que ninguno de los comités organizadores de eventos científicos que se llevan a cabo en Argentina exige el cumplimiento de los requisitos mencionados. También, como hemos señalado, analizamos las exigencias éticas para publicaciones en nuestro país y vimos que son, prácticamente, inexistentes.

Todas estas consideraciones ponen en evidencia la necesidad de establecer algún tipo de resguardo ético en la figura de lo que hemos dado en llamar "Certificado de Conformidad Bioética”. Éste debería formar parte del sistema de calidad interno de cada trabajo científico destinado a la presentación en congresos y publicación en revistas científicas y ser otorgado por un CIEI respaldado por una institu- 
ción académica respetable y con tradición científica.

Proponemos que tales certificados sean requeridos como condición excluyente para presentar y publicar trabajos o recibir auspicios. En otras publicaciones(3) hemos sugerido que la exhibición de los certificados de conformidad bioética sea una condición explícita que conste en las convocatorias, incluso para conferenciantes, panelistas y presentadores.

G) El CIEI como un sistema de calidad. Sellos de acreditación

Una idea en la cual hemos trabajado recientemente se refiere a la posibilidad de observar el proceso de aprobación de los protocolos de investigación clínica sometidos al CIEI como parte de un proceso de generación de calidad, y de incorporar herramientas propias de la moderna gestión de calidad de procesos en la implementación y eventual certificación de protocolos. Nos referimos, específicamente, a sistemas de control tales como la normativas ISO 9000, GMP (buenas prácticas de manufactura y control), GLP (buenas prácticas de laboratorio) y HACCAP (puntos críticos de control de calidad). Utilizadas hasta hace poco para productos y servicios industriales exclusivamente, hemos notado que un número creciente de instituciones de salud, con o sin fines de lucro, comienzan a incorporar estos criterios. Ello nos alienta a abrir una línea de investigación sobre la utilidad directa e indirecta de estas herramientas en la aplicación práctica, cotidiana, de la función del CIEI en el proceso de asegurar calidad en investigación. Entendemos que la aplicación práctica posible es doble. Por un lado, ser parte de las etapas del trazado de procesos; por otro, pensamos que podrían crearse sellos de calidad que identifiquen aquellos estudios que hayan pasado por un CIEI y que hayan sido aprobados sin sugerencias o con sugerencias.
Proponemos la creación del sello épsilon-theta para el primer caso y épsilon para el segundo.

Los investigadores, los equipos de investigación, las instituciones y las empresas que sometan sus estudios a la revisión del CIEI tendrán derecho al uso de dichos sellos que identificarán la calidad ética de los protocolos y agregarán valor al conocimiento y a los productos que, eventualmente, deriven de él. Como se ha dicho, hemos comenzado a trabajar sólo recientemente en estos temas y tenemos un muy interesante camino por recorrer aún en su elaboración intelectual ${ }^{10}$.

\section{Conclusiones}

En Argentina observamos una creciente toma de conciencia-reflejada en la legislaciónsobre la importancia de la inclusión de una instancia de reflexión ética en el ámbito de la salud en términos generales (es el caso de la creación de las comisiones nacionales y algunas provinciales y municipales) y en las instituciones sanitarias en particular (con la incorporación de comités de ética hospitalarios).

Sin embargo, en la legislación no se observa la comprensión de la importancia de la diferenciación entre CEC y CEI ni el discernimiento de sus funciones específicas. Dicha vaguedad podría favorecer algunas formulaciones de las leyes que, aunque bien intencionadas, terminen como enunciados declamatorios, expresiones de deseo o aspiraciones ideales con escasa validez práctica. Los CEI son los menos difundidos y su importancia es fundamental a la hora de proteger a los sujetos de investigación, y garantizar la viabilidad ética de los procedi-

10 Outomuro D, Bortz JE, Kohn Loncarica AG. Estrategias para el Desarrollo y Consolidación de los Comités Independientes de Ética en Investigación en la Argentina. Trabajo presentado a premio en el $4^{\circ}$ Congreso de Bioética de América Latina y el Caribe (FELAIBE). Puerto Rico, septiembre 2003. 
mientos. Pero estimamos que estos comités deben ser independientes.

Hemos aportado datos provenientes de investigaciones realizadas por nuestro grupo en los últimos años para determinar la situación al día de hoy en nuestro país. Asimismo, hemos presentado algunas estrategias que se deben tener en cuenta para desarrollar un plan maestro de implementación de procedimientos éticos en investigación biomédica que, creemos, podrá servir de marco de referencia para nuestros países.

Algunos investigadores consideran como trabas burocráticas las exigencias éticas que hemos sugerido. Suelen apelar al derecho de libertad de investigación. Pero olvidan que la libertad tiene como contrapartida la responsabilidad. Del mismo modo, olvidan que el científico tiene responsabilidad no sólo como tal, sino también como miembro de la sociedad en la que lleva a cabo su tarea. Y en este sentido, como ser social, es mayor, porque actúa desde una posición de privilegio (la sociedad le otorga prestigio y reconocimiento) y asimétrica (es poseedor de un saber que otros no tienen). Mario Heler señala una interesante paradoja:
"La tecnociencia suele ser defendida recurriendo a todos los beneficios que la ciencia ha brindado, brinda y puede seguir brindando. Entonces, la actividad científica posee los méritos por los resultados benéficos. Pero nada tendría que ver con los perjuicios, dado que estos serían resultado de decisiones extrañas. Paradójicamente, no posee responsabilidad sobre los efectos negativos, aunque sus productos los ocasionen y reivindica, simultáneamente, para sí la responsabilidad por la seguridad y el confort obtenidos gracias a sus aplicaciones. La paradoja es una inconsistencia: o bien, es responsable por ambos tipos de consecuencias, o bien, no lo es por ninguno(8)".

La actividad científica como cualquier actividad humana no está eximida de cuestiones éticas. Son los científicos, en tanto personas, y la ciencia, en tanto institución, quienes deben asumir la responsabilidad por las consecuencias, tanto beneficiosas como perjudiciales, que tenga su actividad. El científico, al elegir un curso de acción entre otros, asume el riesgo de dicha elección y si ha tenido libertad al hacerlo ha de responder por las consecuencias de su elección.

\section{Referencias}

1. Mainetti JA. Bioética de la experimentación humana. Experiencia de la Escuela Latinoamericana de Bioética (ELABE). En: Pellegrino Filho A, Macklin R. Investigación en sujetos humanos: experiencia internacional. Santiago de Chile: Programa Regional de Bioética OPS/OMS; 1999: 104.

2. Emanuel E. ¿Qué hace que la investigación clínica sea ética? Siete requisitos éticos. En: Pellegrino Filho A, Macklin R. Investigación en sujetos humanos: experiencia internacional. Santiago de Chile: Programa Regional de Bioética OPS/OMS; 1999: 39.

3. Bortz J, Outomuro D, Sánchez NI, Kohn L. AG. Certificados de conformidad bioética. Revista de la Asociación Médica Argentina 2001; 114 (3): 20-4.

4. Lolas F. Ética de la publicación médica: legalidad y legitimidad. Acta Bioethica 2000; 6 (2): 285.

5. Casino G. Un estudio alerta sobre las falsas expectativas que se generan en los congresos médicos. El País 2002 junio 11; 21. 
Reflexiones sobre el estado actual de la ética en investigación en Argentina - D. Outomuro

6. Lolas F. Temas de Bioética. Santiago de Chile: Editorial Universitaria; 2002: 78.

7. Beauchamp TL, Childress JF, Principles of Biomedical Ethics. New York: Oxford University Press; 1994: 153-157.

8. Heler M. Ética y Ciencia: la responsabilidad del martillo. Buenos Aires: Biblos; 1996: 22. 